\title{
Recent Advances in Large Scale Whole Body MRI Image Analysis - Imiomics
}

\author{
Robin Strand ${ }^{1,2}$, Simon Ekström ${ }^{2}$, Eva Breznik ${ }^{1}$, Therese Sjöholm², Martino Pilia ${ }^{2}$, Lars Lind ${ }^{3}$, Filip \\ Malmberg ${ }^{1,2}$, Håkan Ahlström ${ }^{2}$, Joel Kullberg ${ }^{2}$ \\ ${ }^{1}$ Dept. of Information Technology, Uppsala University \\ ${ }^{2}$ Dept. of Surgical Sciences, Uppsala University \\ ${ }^{3}$ Dept. of Medical Sciences, Uppsala University Uppsala, Sweden \\ Robin.Strand@it.uu.se
}
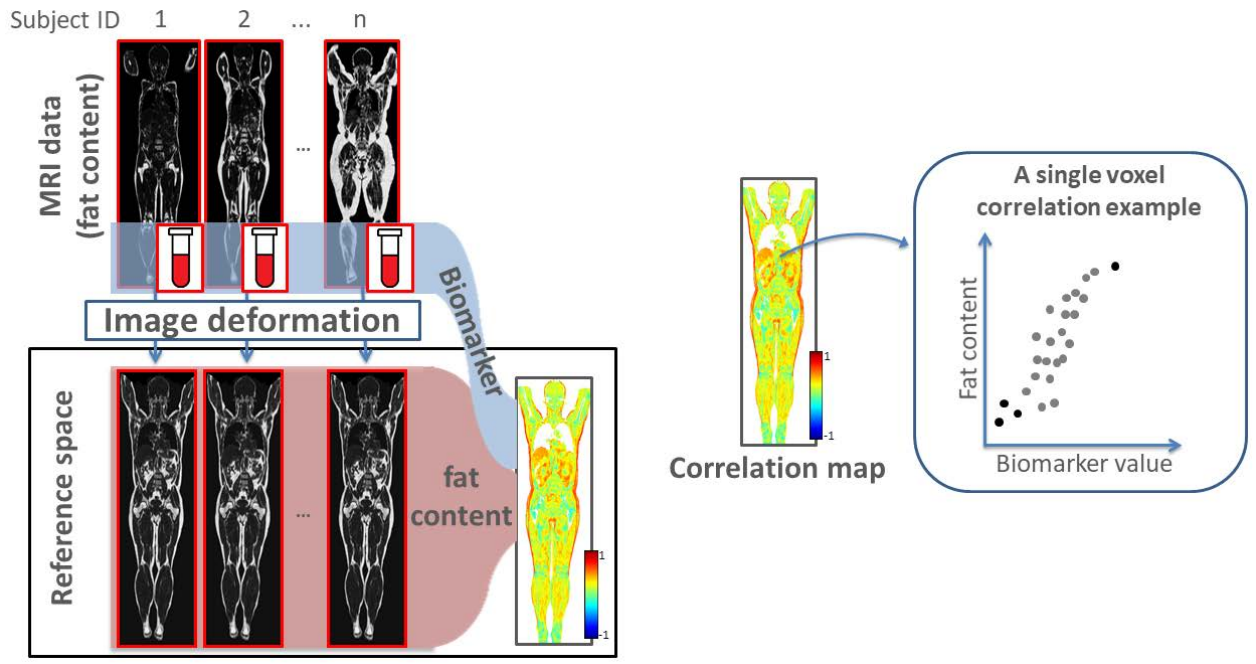

Figure 1: Imiomics concept illustration. In this illustration, the co-aligned images are used for correlation analysis.

\begin{abstract}
Due to the massive amount of medical image data being made available, in research and clinical work, computer-aided tools are valuable and have a great potential for a sustainable work situation for physicians and for generating disease understanding. High-end methods in the present era of big data and artifical intelligence are designed to efficiently find patterns in large scale image data. The amount of data is today often too big to be parsed by human experts, and computer-assisted methods often perform at least as well as human experts on well-defined problems where it is possible to quantify performance by a loss function. This paper gives an overview of a computer-assisted method, Imiomics. Imiomics enables statistical analyses of relations between whole body image image data in large cohorts and other non-imaging data, at an unprecedented spatial resolution. Its usefulness in medicine is illustrated by a number of medical applications, and some aspects
\end{abstract}

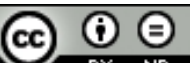

This work is licensed under a Creative Commons Attribution-NoDerivs International 4.0 License.

SIET '20, November 16-17, 2020, Malang, Indonesia

(C) 2020 Copyright held by the owner/author(s).

ACM ISBN 978-1-4503-7605-1/20/09.

https://doi.org/10.1145/3427423.3427465 of technical development that enable the analysis is also presented. We conclude that computer-assisted methods, such as Imiomics, are essential for efficient processing of the huge amount of data in today's medical research and, to some extent, clinical practice.

\section{CCS CONCEPTS}

-Applied computing $\rightarrow$ Imaging; • Computing methodologies $\rightarrow$ Image processing.

\section{KEYWORDS}

Image Processing, Whole Body Imaging, Large Scale Analysis

\section{ACM Reference Format:}

Robin Strand ${ }^{1,2}$, Simon Ekström ${ }^{2}$, Eva Breznik ${ }^{1}$, Therese Sjöholm ${ }^{2}$, Martino Pilia $^{2}$, Lars Lind ${ }^{3}$, Filip Malmberg ${ }^{1,2}$, Håkan Ahlström ${ }^{2}$, Joel Kullberg ${ }^{2} .2020$. Recent Advances in Large Scale Whole Body MRI Image Analysis - Imiomics. In 5th International Conference on Sustainable Information Engineering and Technology (SIET '20), November 16-17, 2020, Malang, Indonesia. ACM, New York, NY, USA, 6 pages. https://doi.org/10.1145/3427423.3427465

\section{INTRODUCTION}

The development in digital image acquisition techniques has in recent years led to an unprecedented availability of image data. For 
example, modern mobile phones have a number of different cameras, and typically at least a dozen programs (apps) for processing and sharing image data. The huge amount of available image data together with better hardware performance has led to an increasing demand on image analysis methods for large scale analysis. Such image analysis methods are often designed to extract patterns in the large scale image data.

Medical image processing has seen a similar development - the amount of data acquired in health care is rapidly increasing. The increasing resolution and available modalities has led to a situation where it is not always feasible for radiologists to manually process all acquired image data [11]. Computer-aided analysis has become an indispensable tool for radiologists' everyday work life [7].

We have developed a medical image analysis concept for statistical analysis of whole body image data, Imiomics (imaging-omics) [15].

Imiomics differs from traditional approaches and concepts in the robust whole body image registration method and in the inherently holistic analysis. Imiomics is holistic in the following three aspects:

- The whole human body is analyzed, as opposed to methods based on analysis of ROIs (regions of interest) where data used in the analysis typically is extracted from pre-defined, pre-segmented regions.

- The image data is used in full resolution in the analysis, as opposed to methods where downsampling is required due to extensive memory requirements such as some deep learning methods.

- Relationships to all other collected non-imaging patient data can be used in the analysis.

Imiomics can be used as a hypothesis free method, for unconditional analysis of relationships between imaging and non-imaging data. Figure 1 shows a conceptual illustration of Imiomics.

The aim of this paper is to survey the recent advances in Imiomics method development and applications in medicine, and to relate Imiomics to sustainability in medicine. This paper gives brief introductions to the technical development on

- image registration, the process of aligning a source image with a reference image by (non-linear) deformation (Sec. 2.2),

- average reference space computation, where the reference image to which all other images are aligned is computed (Sec. 2.3), and

- multiple comparison correction, where the dependence between the millions of statistical tests is taken into consideration and corrected for (Sec. 2.4).

The potential of Imiomics in medicine is illustrated by showing a number of different research applications, including correlation analysis, group comparisons, whole body atlas and anomaly detection, in Sec. 3.

\section{METHODS - TECHNICAL DEVELOPMENT}

\subsection{Image Data}

The data used in this paper is from the Prospective investigation of Obesity, Energy and Metabolism (POEM, http://www.medsci.uu.se/ poem) and from the Epihealth study [9].
The POEM study aims to investigate links between obesity and cardiovascular disorders. It is composed by a sample of 50 -year old inhabitants of Uppsala, Sweden. Ethical approval for the study was obtained from the Regional Ethical Review Board in Uppsala, Sweden (Approval numbers: Uppsala Dnr 2009/057 and Dnr 2012/143), and written consent was obtained from all subjects.

The EpiHealth study provides a resource to study interactions between genotypes and lifestyle factors in a cohort derived from the Swedish population in the age range $45-75$ years regarding development of common degenerative disorders. Ethical approval for the study was obtained from the Regional Ethical Review Board in Uppsala, Sweden. Written informed consent was obtained from all participants.

The inter-subject analysis used in this paper requires absloute image intensity values. All MRI data used in the Imiomics analyses in this paper is whole body fat/water separated MRI, where each voxel holds (absolute) fat percentage values. The imaging protocol and the water-fat image reconstruction method have previously been described, see [2, 8]. In the EpiHealth study, Positron Emission Tomography (PET) data (normalized FDG uptake values, normalized to the mediastinal blood pool) was simultaneously acquired in a PET/MR system.

\subsection{Image Registration}

Three-dimensional image registration enables voxelwise comparisons and analysis and is an essential component in Imiomics. In the image registration process, all whole body images are deformed to a common coordinate system. The registration pipeline utilizes elasticity constraints which are tissue-specific. We build on an assumption that bone is relatively rigid with low inter-subject variation, lean tissue is less rigid with higher inter-individual variation, and adipose tissue is highly elastic with high inter-subject variation. The statistical analysis utilizes the so-obtained point-to-point correspondences.

Figure 1 illustrates how all volume images are deformed to the reference space. This deformation is key in all Imiomics analyses since they build on co-aligned whole body images. An accelerated graph-cut based method for deformable registration has been developed [5].

Formally, the transform that registers a source image $I: \Omega_{S} \rightarrow$ $\mathbb{R}$ with a reference image $I_{R}: \Omega_{R} \rightarrow \mathbb{R}$ is a deformation field $f: \Omega_{R} \rightarrow \Omega_{S}$ that gives a 1-to-1-correspondence between the coordinates in the source and reference images.

The deformation is related to the displacement $u$ as $f(\mathbf{x})=$ $\operatorname{id}(\mathbf{x})+u(\mathbf{x})$.

The energy function, optimized by graph cuts, used in the image registration is on the form

$E(f)=\sum_{p \in \Omega}\left|I_{R}(p)-I(p+u(p))\right|^{2}+\sum_{(p, q) \in \mathcal{N}}\|u(p)-u(q)\|^{2}$.

The sum of squared image intensity differences is used as data term and a diffusion regulariser [12] that penalises high first order derivatives is used as binary regularizing term. Here, $\mathcal{N}$ denotes the set of 6-connected neighbouring voxel pairs in the image.

A key component of the method is to divide the image into overlapping sub-regions and to restrict the optimization to a single sub-region at a time. Since graph-cut optimization does not scale 
linearly in the number of voxels, our proposed speed-up technique reduces the computation time from days to minutes. In essence, the optimization procedure is designed as follows: for a given direction $(( \pm 1,0,0),(0, \pm 1,0),(0,0, \pm 1))$ and for all voxels, compute the unary data term and the spatial binary regularizing term for a small displacement in the given direction. Decide which voxels should move by minimizing the global energy functional. Repeat for all directions. Repeat until convergence.

A proof that the proposed energy functional, restricted to a single small displacement in a given direction, is submodular is presented in [5]. The submodularity ensures an efficient optimization process, by solving a maximum flow/minimum cut problem by graph cuts.

The registration method in [5] was further optimized by moving the matching criterion computation to the GPU [6]. A pipeline model was implemented for efficient overlapping of the criterion computation, data synchronization, and optimization. More general cost functions were also implemented in [6], such as Pearson's correlation coefficient and arbitrary positive exponents in the diffusion reguliser in Eq. 1. This implementation decreased the execution time by a factor of 4 .

See Fig. 2 for an example with different sub-region sizes. We have made the method available as an open source package deform ${ }^{1}$.

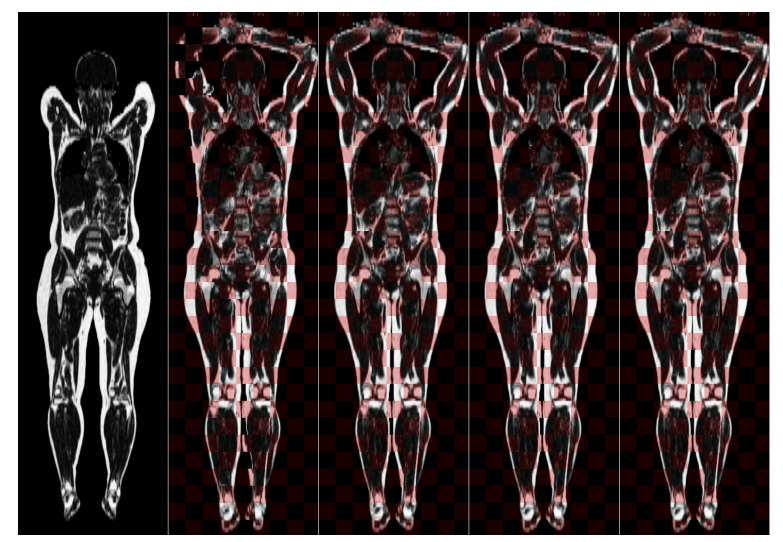

Figure 2: Coronal slices from an example fat percent image. From left to right: unregistered fat percent image checkerboards showing the registered fat images (white) and the reference images (red) for sub-region sizes $1^{3}, 8^{3}, 16^{3}$, and $32^{3}$. Registration when sub-region is set to $32^{3}$ is more than one order of magnitude faster than when sub-region of size $1^{3}$ is used.

\subsection{Average Reference Space Computation}

An essential part of the Imiomics concept is to deform whole body images to align with a reference whole body image, a reference space. As reference space, a whole body image can be chosen from the cohort. In [13], we developed an alternative approach; a method for computing the reference space for a given cohort. This approach aims at computing a reference space that is neutral with respect to

$\overline{{ }^{1} \text { https://github.com/simeks/deform }}$ local tissue volume, i.e., a reference space that is optimal for the image registration.

The aim is to find a new implicit reference $I_{R^{\prime}}: \Omega_{R^{\prime}} \rightarrow \mathbb{R}$ based on a set of $n$ images $\mathcal{I}=\left\{I_{1}, \ldots, I_{n}\right\}$ and an initial reference image $I_{R} \in \mathcal{I}$. The new implicit reference $I_{R^{\prime}}$ is related to $I_{R}$ through a deformation field $d: \Omega_{R} \rightarrow \Omega_{R^{\prime}}$. The deformation that registers $I_{j}$ to $I_{R^{\prime}}$ is given by $f_{j} \circ d^{-1}: \Omega_{R^{\prime}} \rightarrow \Omega_{j}$. The voxel-wise product of Jacobian determinants $J$ of these deformations is given by

$$
\prod_{i=1}^{n} J\left[f_{i} \circ d^{-1}\right]=\prod_{i=1}^{n} J\left[f_{i}\right] \cdot J\left[d^{-1}\right]=\frac{\prod_{i=1}^{n} J\left[f_{i}\right]}{(J[d])^{n}}
$$

where $J[\cdot]$ is the Jacobian determinant. When Eq. 2 equals to 1, there is on average no pointwise volume change. Setting Eq. 2 to 1 leads to

$$
J[d]=\sqrt[n]{\prod_{i=1}^{n} J\left[f_{i}\right]} .
$$

By using an initial reference as starting guess, the proposed method iteratively refines the computed reference by warping it with a deformation that is optimized to achieve a voxel-wise average change of volume associated to the mappings of the images in the cohort equal to zero.

We have made the search-based method used in [13] available as an open-source software ${ }^{2}$ (reimplemented with GPU acceleration).

Figure 3 shows the average Jacobian for the initial reference space and the optimized reference space. Most voxels reach a zero average volume change and due to outliers we observe a relatively high standard deviation.
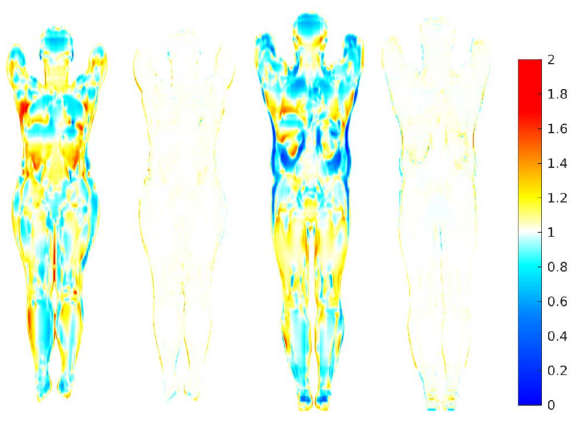

Figure 3: Average Jacobian determinant values for (from left to right) female (initial ref. space), female (optimized ref. space), male (initial ref. space), and male (optimized ref. space). ( $n=167$ for female and $n=159$ for male.)

\subsection{Multiple Comparison Correction}

The voxel-level hypothesis testing in Imiomics suffers from test multiplicity, since the statistical tests at neighboring voxels are in general not independent. Previously published correction methods are mainly developed for neuroimaging and synthetic datasets. We have developed methods for correction of multiple testing in Imiomics whole body analyses [4].

\footnotetext{
${ }^{2}$ https://github.com/m-pilia/disptools
} 


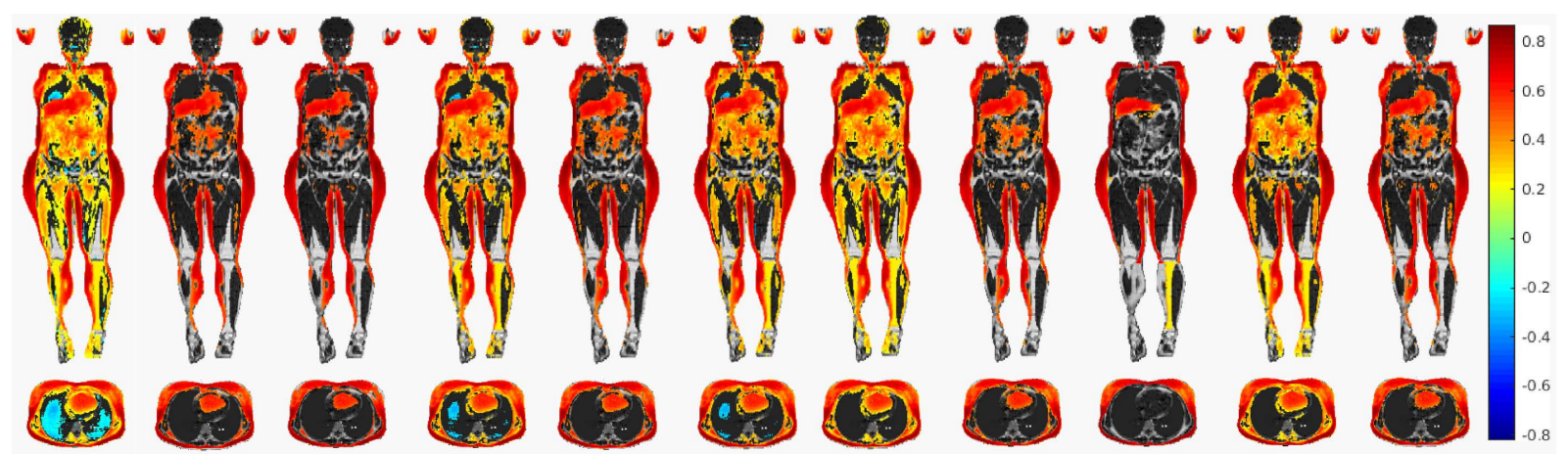

Figure 4: A visual comparison of the effect of multiple test correction methods on BIA measurements vs. local expansion for women. The reference fat content image is shown, overlayed with the original correlation. The voxels deemed significant at significance level $\alpha=0.05$ under the given multiplicity adjustment are color-coded. The color corresponds to the strength of the correlation. From left to right: the original correlation, Holm corrected, RFT voxel- and RFT cluster-wise corrected, permutation-based voxel and clusterextent correction, TFCE corrected, CBA corrected, and finally the anatomy-inclusive corrections by permutation, TFCE, and CBA.

A number of methods were evaluated, controlling the familywise error rate (FWER): Bonferroni type correction, random field theory (RFT), basic permutation-based methods, and threshold-free cluster enhancement (TFCE), as well as the cluster-based analysis (CBA) procedure. The possibility of using anatomical information as a basis for correction was also explored.

Figure 4 shows coronal and axial slices of voxel-wise correlations between the local expansion (by the Jacobian determinant) and total fat mass, using different correction methods. The total fat mass is measured by bioimpedance analysis (BIA).

As concluded by Breznik et al. [4], the recommended choice of methods for correction for multiplicity for whole body MRI analyses are the correction methods that rely on pre-segmented organs. In other words the anatomy-inclusive corrections by permutation, TFCE, and CBA, see Fig. 4. If a segmented reference image is not available, the original TFCE is preferable.

\section{MEDICAL APPLICATIONS}

\subsection{Correlation Analysis - Adipose and Lean Tissue Distribution}

In a "proof-of-principle" study, it was shown how Imiomics could visualise the association between fat/lean tissue and local tissue volume/fat content. Imiomics analyses were performed on total fat mass and total lean mass measured by dual energy $\mathrm{x}$-ray absorptiometry (DXA), as well as waist-hip-ratio. Fat mass and distribution measures were found to be associated to the detailed differences in both tissue volume and fat concentration throughout the body. Positive correlatation $(\mathrm{r}>0.50, \mathrm{p}<0.05)$ between fat mass and tissue volume in all subcutaneous areas of the body was found. There was also a positive correlation between fat mass and volumes of intraperitoneal fat, retroperitoneal fat, perirenal fat and the liver. Negatively to lung volume was found. Fat mass also correlated positively with volumes of paravertebral muscles, and muscles in the ventral part of the thigh and lower limb. Distinct correlation between fat mass and fat content in subcutaneous adipose tissue at the trunk was found. Lean mass was positively related to the large skeletal muscles and the skeleton.

Figure 5 shows coronal slices of correlation maps of how tissue volume correlates to total fat mass, total lean mass and waist-hipratio.

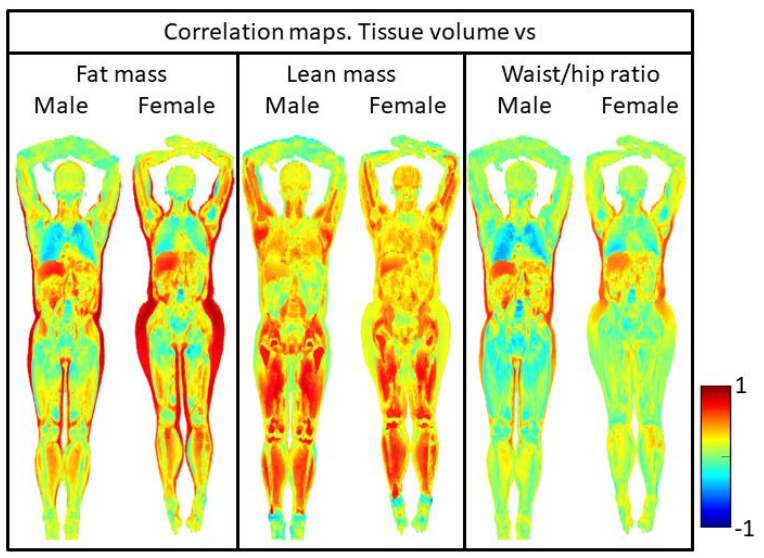

Figure 5: Imiomics analysis results on local tissue volume. Curved coronal slices of the full volume images are shown. Correlation values ( $r$, Pearson coefficient) of the non-imaging parameters total fat mass (as measured by DXA, left), total lean mass (measured by DXA, middle), and waist/hip ratio (right) vs, local tissue volume (Jacobian determinant of the deformation field) are shown color-coded.

\subsection{Group Comparisons - The Metabolic Syndrome and its Components}

Imiomics group comparisons were used in [10] to investigate the relationship between occurence of the metabolic syndrome and body composition. 


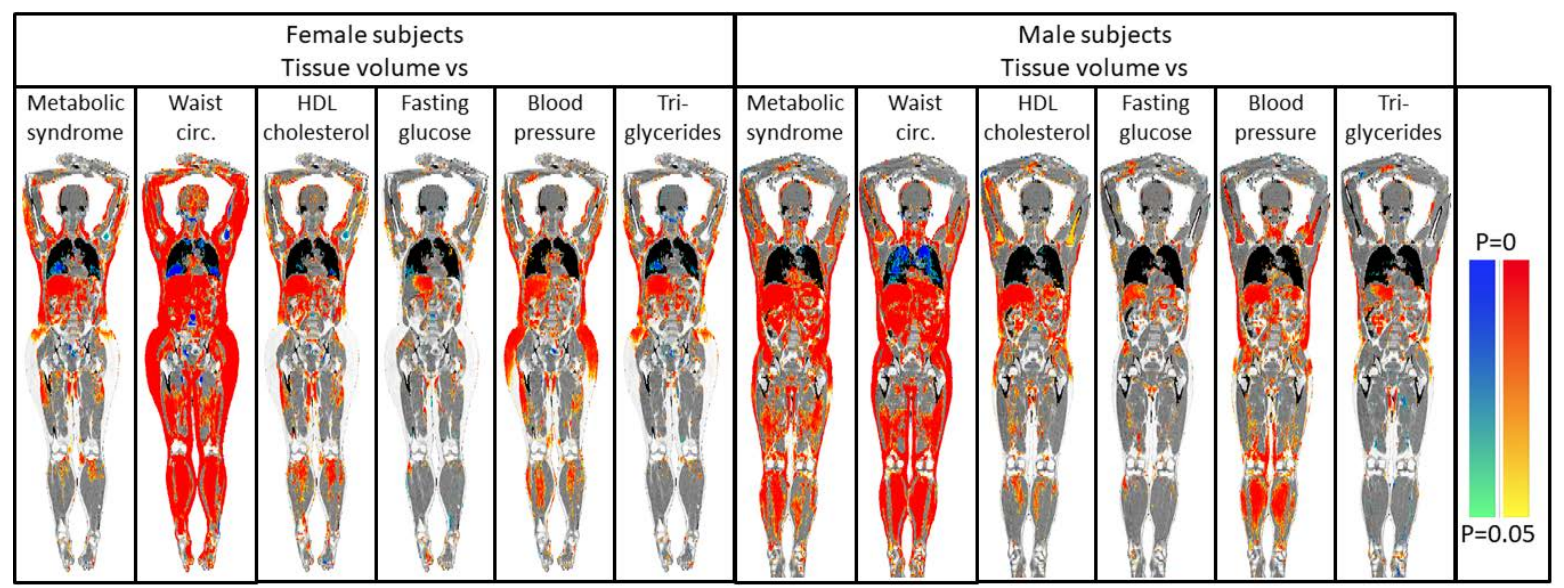

Figure 6: Imiomics analysis results on local tissue volume vs. Metabolic syndrome. The analyses are based on group comparisons and the corresponding p-value, and the sign of the association is color-coded. Positive relations are shown in yellow-red and negative relations in green-blue. Voxels with $P$-values above 0.05 are transparent and shows the fat/water-content intensity values.

The five conditions described below are metabolic risk factors. These risk factors tend to occur together. A person must have at least three metabolic risk factors to be diagnosed with the metabolic syndrome.

The Metabolic syndrome is based on the five following components: waist circumference, high-density lipoprotein (HDL) cholesterol, glucose, blood pressure, and triglycerides. A person who has non-normal high values in three of the five components has the Metabolic syndrome [1].

For each of the components, and for the Metabolic syndrome, an Imiomics group comparison analys was performed. This resulted in whole body maps with pointwise relations between each of the components and the Metabolic syndrome, see Fig. 6.

The voxel-wise analysis found relations between the metabolic syndrome and liver, heart, and perirenal fat volume. Also, sex differences in associations with adipose tissue in the subcutaneous region were identified. In women, there were regional differences in associations to adipose tissue volumes in upper versus lower body, and dorsal versus ventral abdominal depots in the Metabolic syndrome p-maps. See [10] for a thorugh analysis on the metabolic syndrome.

\subsection{Whole Body Atlas and Anomaly Detection - Lymphoma}

The Imiomics approach has also been used for anomaly detection in lymphoma [14]. In this work PET/MR hybrid imaging data was used in the analyses. A multimodality whole body normal atlas of functional 18F-fluorodeoxyglucose (FDG) PET and anatomical fat/water-separated MR data was generated. For subjects with suspected pathology, pointwise statistical tests were performed to detect deviations from the normal atlas. The following additional criteria were used to minimize the number of false positive detected pathologies:

- Non-normal regions' diameter should be at least $10 \mathrm{~mm}$.
- Non-normal regions' mean FDG uptake should be larger than the mean liver FDG uptake.

Figure 7 shows an example anomaly detection in a suspected lymphoma.
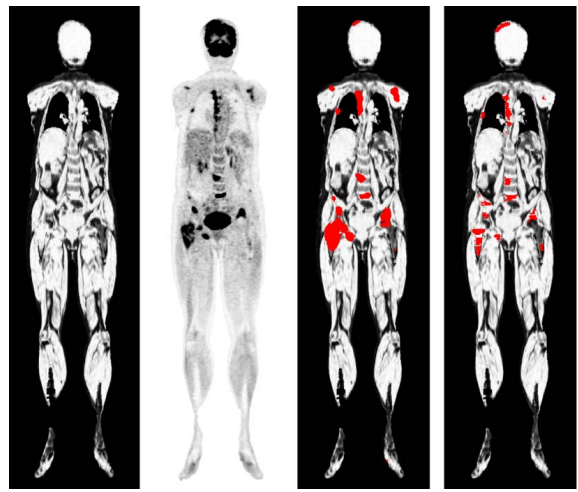

Figure 7: FDG anomaly detection task performed for a female subject with suspected lymphoma. A representative coronal slice is shown of the registered water fraction and normalised FDG uptake images, and of the automatically detected and manually segmented tumours. Detected and segmented lesions are displayed in red and overlaid on the water fraction image.

\section{DISCUSSION AND CONCLUSIONS}

Computer-supported methods that can detect patterns in huge amounts of data, such as Imiomics, have the potential to increase efficacy and efficiency in clinical care. This holds in particular for methods in the category of Artificial Intelligence (AI), i.e., methods that can emulate some kind of (human) intelligence. The development of imaging acquisition techniques has enabled increasing 
resolution (i.e. increasing amount of data) in radiology exams, and radiological imaging data grows at a disproportionate rate. Also, to provide health care for the increasing population is demanding. We argue that Imiomics, which can compute statistical relationships between large scale whole body image data and other biomarkers at a very high spatial resolution, and other methods for computer assisted image processing which can detect patterns in large scale data, will be crucial for maintaining a sustainable health care. In addition, the Imiomics examples shown in this paper suggest that computer assisted image processing in medicine has the potential to improve health care by detecting pathology (patterns) which are not detectable by the human eye.

Imiomics can also be used to generate hypothesis by the unconditional analysis of relationships between imaging and non-imaging data. McDonald et al. concluded in 2015 that a typical radiologist must interpret one image every 3-4 seconds over the span of a workday to satisfy workload demands [11] - radiologists can not analyze all the massive amount of data collected, with any reasonable work load. In the present era of increasing amount of data, the decline in image reimbursements and the limited number of trained radiologists, computer-aided analysis tools are essential for a sustainable situation for physicians $[3,11]$. An integrated AI component in the imaging workflow has the potential to increase efficiency, reduce errors and achieve objectives with limited manual input [7].

Improved Analysis of Image Data. Imiomics can be used in several medical applications:

- Correlation analysis (Sec. 3.1) In this paper, Imiomics correlation analyses is exemplified by a fat and lean tissue distribution application in Sec. 3.1. Imiomics correlation analyses have many additional potential applications. One valuable application in big data analysis is hypothesis generation, where new/unknown associations between a large number of biomarkers and imaging biomarkers can be detected. This was recently applied to Proteomics-Imiomics analyses, and see great potential in Genomics-Imiomics analyses.

- Group analysis (Sec. 3.2) Group comparison analysis allows comparison of groups obtained by, for example, thresholding ordinal or continuous variables/biomarkers or by categorical variables/biomarkers.

- Statistical atlas creation (Sec. 3.3) Statistical atlas creation can be used to represent normality in a cohort. This statistical atlas of normality can be used to detect particularities in different cohorts or sub-cohorts.

- Anomaly detection (Sec. 3.3) Different anomalies can be detected by comparing new images with the statistical atlas, for example, point-wise, tissue-wise, or organ-wise.

- Longitudinal analysis (not included in this paper, see [15]) Examples of longitudinal analyses include disease progression, drug efficacy and e.g. weight loss where this technology allows visualization of changes.

All of these applications can answer questions such as "How does a particular disease manifest in image data", where image data refers to fat or lean tissue content, tissue volume and PET tracer uptake in the examples shown in this paper.
In summary, Imiomics and other computer assisted image processing methods can be used to enable increased disease understanding and automatic disease/pathology detection, which potentially leads to correct diagnosis, and fewer missed diagnoses.

\section{REFERENCES}

[1] KGMM Alberti, Robert H Eckel, Scott M Grundy, Paul Z Zimmet, James I Cleeman, Karen A Donato, Jean-Charles Fruchart, W Philip T James, Catherine M Loria, and Sidney C Smith Jr. 2009. Harmonizing the metabolic syndrome: a joint interim statement of the international diabetes federation task force on epidemiology and prevention; national heart, lung, and blood institute; American heart association; world heart federation; international atherosclerosis society; and international association for the study of obesity. Circulation 120, 16 (2009), 1640-1645.

[2] Johan Berglund, Lars Johansson, Håkan Ahlström, and Joel Kullberg. 2010. Threepoint Dixon method enables whole-body water and fat imaging of obese subjects. Magnetic Resonance in Medicine: An Official fournal of the International Society for Magnetic Resonance in Medicine 63, 6 (2010), 1659-1668.

[3] Giles WL Boland, Alexander S Guimaraes, and Peter R Mueller. 2009. The radiologist's conundrum: benefits and costs of increasing CT capacity and utilization. European radiology 19, 1 (2009), 9-11.

[4] Eva Breznik, Filip Malmberg, Joel Kullberg, Håkan Ahlström, and Robin Strand. 2020. Multiple comparison correction methods for whole-body magnetic resonance imaging. Fournal of Medical Imaging 7, 1 (2020), 014005.

[5] Simon Ekström, Filip Malmberg, Håkan Ahlström, Joel Kullberg, and Robin Strand. 2020. Fast graph-cut based optimization for practical dense deformable registration of volume images. Computerized Medical Imaging and Graphics (2020), 101745.

[6] Simon Ekström, Martino Pilia, Joel Kullberg, Håkan Ahlström, Robin Strand, and Filip Malmberg. 2020. Faster dense deformable image registration by utilizing both CPU and GPU. Unpublished manuscript (2020).

[7] Ahmed Hosny, Chintan Parmar, John Quackenbush, Lawrence H Schwartz, and Hugo JWL Aerts. 2018. Artificial intelligence in radiology. Nature Reviews Cancer 18,8 (2018), 500-510.

[8] Joel Kullberg, Lars Johansson, Håkan Ahlström, Frederic Courivaud, Peter Koken, Holger Eggers, and Peter Börnert. 2009. Automated assessment of whole-body adipose tissue depots from continuously moving bed MRI: a feasibility study. Journal of Magnetic Resonance Imaging: An Official Journal of the International Society for Magnetic Resonance in Medicine 30, 1 (2009), 185-193.

[9] Lars Lind, Sölve Elmståhl, Ebba Bergman, Martin Englund, Eva Lindberg, Karl Michaelsson, Peter M Nilsson, and Johan Sundström. 2013. EpiHealth: a large population-based cohort study for investigation of gene-lifestyle interactions in the pathogenesis of common diseases. European journal of epidemiology 28, 2 (2013), 189-197.

[10] Lars Lind, Robin Strand, Karl Michaëlsson, Håkan Ahlström, and Joel Kullberg. 2020. Voxel-wise Study of Cohort Associations in Whole-Body MRI: Application in Metabolic Syndrome and Its Components. Radiology 294, 3 (2020), 559-567.

[11] Robert J McDonald, Kara M Schwartz, Laurence J Eckel, Felix E Diehn, Christopher H Hunt, Brian J Bartholmai, Bradley J Erickson, and David F Kallmes. 2015. The effects of changes in utilization and technological advancements of crosssectional imaging on radiologist workload. Academic radiology 22, 9 (2015), 1191-1198.

[12] Jan Modersitzki. 2004. Numerical Methods for Image Registration. Oxford University Press.

[13] Martino Pilia, Joel Kullberg, Håkan Ahlström, Filip Malmberg, Simon Ekström, and Robin Strand. 2019. Average volume reference space for large scale registration of whole-body magnetic resonance images. PloS one 14, 10 (2019).

[14] Therese Sjöholm, Simon Ekström, Robin Strand, Håkan Ahlström, Lars Lind, Filip Malmberg, and Joel Kullberg. 2019. A whole-body FDG PET/MR atlas for multiparametric voxel-based analysis. Scientific reports 9, 1 (2019), 1-10.

[15] Robin Strand, Filip Malmberg, Lars Johansson, Lars Lind, Magnus Sundbom, Håkan Ahlström, and Joel Kullberg. 2017. A concept for holistic whole body MRI data analysis, Imiomics. PloS one 12, 2 (2017). 